\title{
Achenbach syndrome
}

\author{
Kaori Notomi MD, Taku Harada MD
}

Cite as: CMAJ 2019 May 27;191:E584. doi: 10.1503/cmaj.181293

A previously healthy 40-year-old man presented to the emergency department with sudden onset of swelling and changes in colour and sensation in his right middle finger. On physical examination, we found a subcutaneous hematoma and swelling, with normal capillary refill and sensation (Figure 1A). Results from laboratory tests showed normal platelet counts and coagulation function. The hematoma and associated symptoms had disappeared by the 1-week follow-up (Figure 1B). We diagnosed Achenbach syndrome.

Achenbach syndrome is usually observed in women aged 40 years or older, and its cause is unknown. ${ }^{1}$ It involves a sudden abnormal sensation (pain, numbness or stiffness) in the fingers and palms, despite the absence of obvious causes such as trauma or coagulopathy; a hematoma forms at the symptom site. ${ }^{2}$ The locations most likely to be affected are the middle and base regions of the second or middle finger. ${ }^{1}$

The differential diagnosis for Achenbach syndrome is diverse., ${ }^{2,3}$ The most urgent alternative would be acute limb ischemia, which would present with sudden onset of poorly demarcated pain with distal coldness and blue discolouration. Other possible diagnoses include Raynaud phenomenon, Buerger disease, acrocyanosis, Gardner-Diamond syndrome and frostbite, ${ }^{2,3}$ supported by a relevant history that may include arteriosclerosis, systemic symptoms exposure to cold and trauma. ${ }^{3}$ In the absence of other diagnoses, the only investigations that are necessary are a blood count, coagulation function, immunological assay and inflammatory markers (sedimentation rate and C-reactive protein). ${ }^{2}$ With rest and cooling, the condition should resolve without sequelae after several days. ${ }^{2}$

A prompt diagnosis helps address patient concerns and avoids unnecessary referrals and invasive investigations. Achenbach syndrome may recur.

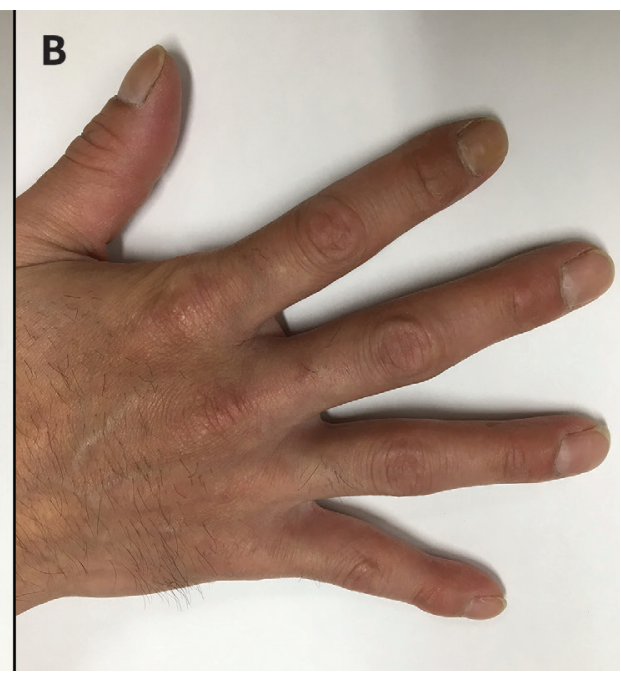

Figure 1: (A) Subcutaneous hematoma and swelling in the right middle finger of a 40-year-old man with sudden-onset colour and sensation change in the finger (initial presentation). (B) Absence of subcutaneous hematoma 7 days after the patient's emergency department visit.

\section{References}

1. Carpentier PH, Maricq HR, Biro C, et al. Paroxysmal finger haematoma - a benign acrosyndrome occurring in middle-aged women. Vasa 2016;45: 47-62.

2. Kordzadeh A, Caine PL, Jonas A, et al. Is Achenbach's syndrome a surgical emergency? A systematic review. Eur J Trauma Emerg Surg 2016;42:439-43.

3. Brown PJ, Zirwas MJ, English JC III. The purple digit: an algorithmic approach to diagnosis. Am J Clin Dermatol 2010;11:103-16.

\section{Competing interests: None declared.}

This article has been peer reviewed.

The authors have obtained patient consent.

Affiliations: Division of General Medicine (Notomi, Harada), Showa Medical University Hospital, Shinagawa-ku, Tokyo, Japan; Diagnostic and Generalist Medicine (Harada), Dokkyo Medical University Hospital, Shimotsuga-gun, Tochigi, Japan

Correspondence to: Taku Harada, hrdtaku@gmail.com 